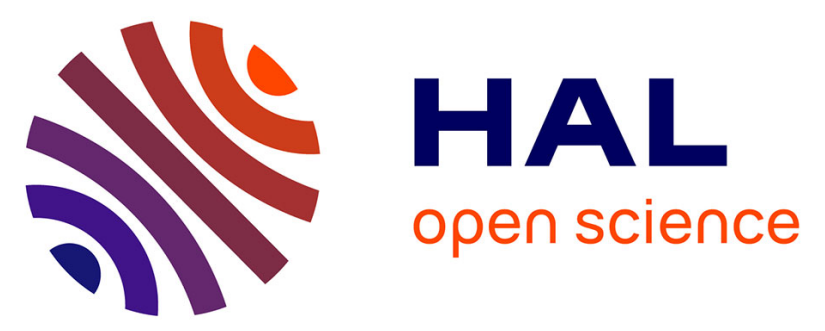

\title{
Vehicles Platooning in Urban Environments: Integrated Consensus-based Longitudinal Control with Gap Closure Maneuvering and Collision Avoidance Capabilities
}

\author{
Ahmed Khalifa, Olivier Kermorgant, Salvador Dominguez, Philippe Martinet
}

\section{- To cite this version:}

Ahmed Khalifa, Olivier Kermorgant, Salvador Dominguez, Philippe Martinet. Vehicles Platooning in Urban Environments: Integrated Consensus-based Longitudinal Control with Gap Closure Maneuvering and Collision Avoidance Capabilities. ECC19 - European Control Conference, Jun 2019, Naples, Italy. pp.1695-1701. hal-02057396

\section{HAL Id: hal-02057396 https://hal.science/hal-02057396}

Submitted on 5 Mar 2019

HAL is a multi-disciplinary open access archive for the deposit and dissemination of scientific research documents, whether they are published or not. The documents may come from teaching and research institutions in France or abroad, or from public or private research centers.
L'archive ouverte pluridisciplinaire HAL, est destinée au dépôt et à la diffusion de documents scientifiques de niveau recherche, publiés ou non, émanant des établissements d'enseignement et de recherche français ou étrangers, des laboratoires publics ou privés. 


\title{
Vehicles Platooning in Urban Environments: Integrated Consensus-based Longitudinal Control with Gap Closure Maneuvering and Collision Avoidance Capabilities
}

\author{
Ahmed Khalifa, Olivier Kermorgant, Salvador Dominguez, and Philippe Martinet
}

\begin{abstract}
This paper proposes a distributed longitudinal controller for car-like vehicles platooning that travel in an urban environment. The presented control strategy combines the platoon maintaining, gap closure, and collision avoidance functionality into a unified control law. A consensus-based controller designed in the path coordinates is the basis of the proposed control strategy and its role is to achieve position and velocity consensus among the platoon members taking into consideration the nature of the motion in an urban environment. For platoon creation, gap closure scenario is highly recommended for achieving a fast convergence of the platoon. For that, an algorithm is proposed to adjust the controller parameters online. A longitudinal collision between followers can occur due to several circumstances. Therefore, the proposed control strategy considers the assurance of collision avoidance by the guarantee of a minimum safe inter-vehicle distance. Convergence of the proposed algorithm is proved in the different modes of operations. Finally, studies are conducted to demonstrate and validate the efficiency of the proposed control strategy under different driving conditions. To better emulate a realistic setup, the controller is tested by an implementation of the car-like vehicles platoon in a vehicular mobility simulator called ICARS, which considers the real vehicle dynamics and other platooning staff in urban environments.
\end{abstract}

\section{INTRODUCTION}

The car-like vehicles platooning in urban environments is one of the current trends to solve the problem of the cars redistribution in the car sharing system. This is achieved by utilizing vehicle platoon guided by professional drivers who come to pick up and drop off vehicles over the stations [1].

In the literature, variety of platoon models are presented including linear and nonlinear models. Based on different assumptions, three linear models are used including single integrator model [2], double integrator model [3], and third order model [4]. Few studies have addressed the problem of vehicles platoon in an urban environment, e.g., [5]. In such studies, the authors uses a first order model of the platoon which is widely different from the real vehicle dynamics. In our previous research [1], a second order dynamic model is utilized and transformation to its corresponding one in the path coordinates is given so that we can design

The authors are with the Laboratoire des Sciences du Numérique de Nantes (LS2N), Ecole Centrale de Nantes, 1 rue de la Noë, 44321 Nantes, France, given-name.namedec-nantes.fr.

A. Khalifa is on leave from the Department of Industrial Electronics and Control Engineering, Faculty of Electronic Engineering, Menoufia University, Egypt.

P. Martinet is with the INRIA Sophia Antipolis, Sophia Antipolis, France. a controller to satisfy control objectives defined in the operational coordinates.

Adaptive Cruise Control (ACC) is presented as the simplest platoon controller, and it is role is to maintain a safe distance with the preceding vehicle. Recently, platoon control is designed based on a distributed controller known as Cooperative Adaptive Cruise Control (CACC) [6] which needs information from the neighbor vehicles to achieve a global consensus. Six main approaches are presented to design a platoon controller including Linear [4], Optimal [7], $\mathcal{H}_{\infty}$-based [8], Sliding Mode [9], Model predictive [10], and Consensus-based controller [3]. However, none of these works tackles the control in the Curvilinear coordinates. Furthermore, they assume that the leader navigates with constant velocity which is not feasible to the motion in urban environments in which it travels with variable velocities. Therefore, in our previous research [1], the authors propose a controller that can achieve asymptotic stability of the tracking errors and the platoon string stability considering variable velocity of leader.

For platoon creation, gap closure scenario, between a merging vehicle and an in-platoon vehicle or at the end of a splitting scenario, is required. Hence, a vehicle should be able to cooperatively close or open a gap. The gap closure requirement is to increase the response speed in order to decrease the gap closing time. In other words, at large inter-vehicular distance, a reduced damping can be desirable to allow significant velocity differences for gap closing performance. Therefore, in this research, platooning and gap closing functionality is combined into a single control design.

Recently, control problems with collision avoidance have got large progress. Many approaches have been developed in this field. Firstly, Rule-based approach (e.g., Artificial Potential Field [11]). Secondly, optimization-based approach (e.g., Model Predictive Control [10]). Thirdly, with the aid of time-optimal path-constrained trajectory planning techniques, for instance, the authors in [12] present a trajectory planning algorithm to generate time optimal velocity profiles, which assures the rear-end collision avoidance and considers both velocity and acceleration constraints for the leader and follower vehicles. In this approach, the problem is handled at the path generation level, i.e., the system has a control on the path of the leader and followers. However, this is not the case for our application in which the followers follow the path generated from the leader 
that has a free motion. Fourthly, in [13], the vehicles are equipped with a collision detection and avoidance module, like pedestrians crossing the road or other vehicles, at the low level controller. Consensus-based controller with collision avoidance capability applied to quad-rotors formation control is introduced in [14] in which the authors propose collision avoidance strategy based on the potential field method. However, they use a potential function that has a switching effect (i.e., the transition from the non-collision case to collision case and vice versa is discontinuous). In our study, the platoon longitudinal controller is supported by a collision avoidance capability so that a safe inter-vehicle distance, in case of abnormal situation occurs such as sudden braking of a vehicle inside the platoon, can be guaranteed. Therefore, the main high-level longitudinal control law is modified in order to make the platoon able to guarantee a safety region to keep a minimum inter-vehicle safe distance and to avoid the collision. Moreover, we use a potential function that has smooth and local effect during the transitions.

The proposed controller is implemented in a home made fast prototyping environment called ICARS that is used for simulation. It is developed under Robot Operating System (ROS) and it has features such as real vehicle dynamics and models (engine, transmission, braking systems, etc.), both low and high level measurements units with noises, low level communication module between different parts of the vehicle and the on-board computer, real maps and urban environments with different road conditions (curvature, bumps, etc.), vehicle communication modules, and fully compatible with embedded computer architecture.

Summarizing, the main contributions of this research are,

- design of a gap closure algorithm;

- design of a longitudinal anti-collision strategy which guarantees a safety zone between the vehicles;

- combining platooning, gap closure, collision avoidance functionalities into a single control law;

- proof of the system convergence.

\section{PROBLEM STATEMENT}

\section{A. Kinematic Model of Car-like Vehicle}

The kinematic model of a car-like vehicle can be simplified to that of a bicycle model [1], see Fig. 1, in which the left and right wheels are considered as a single wheel at the center of front and rear axles.

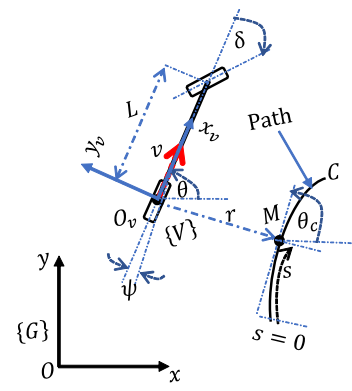

Fig. 1: Kinematics of car-like vehicle.
1) Cartesian Coordinates: Let $\{V\}, O_{v}-x_{v} y_{v}$, represents the vehicle body-fixed reference frame with its $x$-axis is in the vehicle forward direction and its origin is at the center of its rear axle, $O_{v}$, see Fig. 1. The vehicle configuration, with respect to the world-fixed inertial reference frame, $\{G\}, O-x y$, is represented by the generalized coordinates $p=\left[\begin{array}{lll}x & y & \theta\end{array}\right] \in \mathbb{R}^{3}$, where $\left[\begin{array}{ll}x & y\end{array}\right]^{T}$ is the position and $\theta$ represents its orientation. The vehicle steering angle is given by $\delta$. Assuming that the wheels cannot slip sideways, the vehicle velocity in the body $x$-direction is $v$, and it is zero in the body $y$-direction. The front wheel is mounted at a distance $L$ from the rear wheel. The kinematic model is given by

$$
\begin{aligned}
\dot{x} & =v \cos (\theta), \\
\dot{y} & =v \sin (\theta), \\
\dot{\theta} & =\frac{v}{L} \tan (\delta) .
\end{aligned}
$$

2) Curvilinear Coordinates: The vehicle kinematics can be represented in the Curvilinear coordinates $(s \quad r \psi)^{T}$, as illustrated in Fig. 1. The path to be tracked is $C$. The path length $s$ (Curvilinear abscissa) is defined between the closest point $M$ to $O_{v}$. The angular deviation of the vehicle with respect to the path is resented by $\psi=\theta-\theta_{c}$, where $\theta_{c}$ is the angle between path tangent at $M$ and the $x$-axis. The lateral deviation, $r$, is defined as the signed orthogonal distance from the rear axle center, $O_{v}$, to point $M$.

Given the geometry in Fig. 1, the kinematic model in the path coordinates is given as

$$
\begin{aligned}
\dot{s} & =v \frac{\cos (\psi)}{1-r \kappa(s)}, \\
\dot{r} & =v \sin (\psi), \\
\dot{\psi} & =v\left(\frac{\tan (\delta)}{L}-\frac{\kappa(s) \cos (\psi)}{1-r \kappa(s)}\right),
\end{aligned}
$$

where $\kappa(s)$ is the path curvature at point $M$.

\section{B. Longitudinal Dynamics}

The vehicle longitudinal dynamics are affected by many factors such as the engine, drive line, brake system, aerodynamics drag, tire friction, rolling distance, and gravitational force, etc [9]. For the vehicle dynamics, let us assume:

Assumption 1. The body is rigid and symmetric.

Assumption 2. The throttle and brake inputs are combined as one control input.

Assumption 3. The effect of pitch and yaw motions is neglected.

Therefore, the longitudinal dynamic equation can be represented by

$$
M_{v} a+C_{v} v+G_{v}=F_{v}
$$

where $a=\dot{v}$ denotes the vehicle acceleration in the body frame, and $M_{v}, C_{v}, G_{v}$, and $F_{v} \in \mathbb{R}$ are terms representing 
the vehicle Inertia, Coriolis and Gravity effects, and input force, respectively.

For the purpose of longitudinal HLC, The longitudinal LLC is considered as an inverse model-based control law which is given by

$$
F_{v}=M_{v} \mu+C_{v} v+G_{v}
$$

where $\mu$ is the new input signal to be designed.

Therefore, the vehicle linear longitudinal dynamic model is given as

$$
\eta=\mu
$$

Since we aim at platooning in an urban environment, relation between the vehicle dynamics in the Curvilinear and Cartesian coordinates will be given as follows.

Let us rewrite $(2 a)$ as

$$
\dot{s}=J v
$$

Thus, the vehicle acceleration in the Curvilinear coordinates, $\eta$, is given as

$$
\eta=J a+\dot{J} v
$$

where $\eta=\dot{q}=\ddot{s}$ and $q=\dot{s}$ are the vehicle acceleration and velocity in the path coordinates respectively.

Let us denote the control signal in the path coordinates as $u$. If the relation between $u$ and $\mu$ is given by

$$
\mu=\frac{1}{J}(u-\dot{J} v)
$$

then, the path coordinates longitudinal dynamics are given by

$$
\begin{aligned}
& \dot{s}=q, \\
& \dot{q}=\eta=u .
\end{aligned}
$$

\section{Platoon Longitudinal Model}

Consider $N+1$ platoon vehicles as illustrated in Fig. 2, which consists of a leading vehicle (called as the leader with index 0 ) and $N$ following vehicles (called followers with index $i ; i=1,2, \ldots, N$ ). The platoon runs on urban and flat road, and the vehicles can share information by either sensor-based or communication-based way.

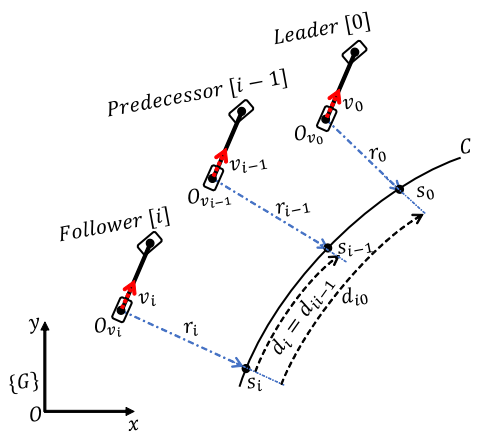

Fig. 2: Car-like vehicle platoon representation.

Since the vehicles considered in the car sharing system are small passenger cars, one can assume that they have equal dynamics. The in-platoon vehicle dynamic model in the Curvilinear coordinates is given by

$$
\begin{aligned}
& \dot{s}_{i}=q_{i}, \\
& \dot{q}_{i}=u_{i} .
\end{aligned}
$$

For vehicles string, let us define the inter-vehicle distance as $d_{i}=s_{j}-s_{i}$ that is the actual relative Curvilinear distance between vehicle $i$ and its predecessor $j=i-1$. In addition, the desired inter-vehicle distance between vehicles $i$ and $j$ is represented as $d_{r, i}$. Many spacing policies are proposed in the literature [15]. For higher traffic flow, the constant spacing policy method is utilized in our research.

\section{Control Objectives}

The platoon can navigate in an urban environment/Curvilinear path and track a leading vehicle that is either autonomous/human driven, and each follower vehicle, $i$, can receive leader position, velocity, and acceleration represented by $s_{0}, q_{0}, \eta_{0}$ respectively and predecessor position, $s_{i-1}$. Under these circumstances, the objective is to combine platooning, gap closing, and collision avoidance functionality into a single control design. Therefore, we aim at achieving the following goals:

- convergence of the tracking errors as well as string stability;

- gap closure requirement: to increase the response speed in order to decrease the gap closing time for large intervehicle distances;

- collision avoidance: to guarantee a safety region between the vehicles (keep a minimum safe intervehicle distance in case of an abnormal situation).

\section{PLATOON CONTROL DESIGN}

Let $\mathcal{G}=(\mathcal{V}, \mathcal{E}, \mathcal{M})$ be a digraph describing the information flow topology among the in-platoon vehicles. The graph has set of nodes $\mathcal{V}=1, \ldots, N$ and set of edge $\mathcal{E} \subseteq \mathcal{V} \times \mathcal{V}$. Each graph node represents a vehicle whose dynamics are defined by (10). Let $\mathcal{M}=\left[m_{i j}\right]_{N \times N}$ be adjacency matrix associated with $\mathcal{G}$. Its elements are defined as follows: if vehicle $i$ can obtain information from vehicle $j$, then $m_{i j}=1$; otherwise $m_{i j}=0$. Therefore, it represents graph topology. An augmented digraph $\overline{\mathcal{G}}$ is also defined in order to involve the leader in the network.

We propose a control law given by

$$
u_{i}=u_{i}^{p}+u_{i}^{c},
$$

where $u_{i}^{p}$ is the part of the control signal in charge of the platoon creation and maintaining, while $u_{i}^{c}$ is responsible for the collision avoidance.

\section{A. Design of $u_{i}^{p}$}

$$
u_{i}^{p}=\eta_{0}+b\left(q_{0}-q_{i}\right)+\sum_{j=0}^{N} k_{i j} m_{i j}\left(s_{j}-s_{i}-d_{r, i j}\right),
$$

where, $b$ and $k_{i j}$ are tuning parameters, and $d_{r, i j}$ is the desired inter-vehicle distance between vehicles $i$ and $j$. 
Since we assume a PLF topology, (12) can be reformulated as

$$
u_{i}^{p}=\eta_{0}+b e_{q, i 0}+k_{0} e_{s, i 0}+k_{1} e_{s, i},
$$

where $e_{s, i 0}=s_{0}-s_{i}-d_{r, i 0}, e_{q, i 0}=q_{0}-q_{i}, e_{s, i}=s_{j}-$ $s_{i}-d_{r, i}$, and $k_{0}$ and $k_{1}$ are controller parameters.

1) Internal Stability Analysis: If the control law (13) is applied to the platoon system (10), and defining the intervehicle distance error, $e_{s, i}$, in terms of the errors with respect to leader, $e_{s, i 0}$, as $e_{s, i}=s_{j}-s_{i}-d_{r, i}=e_{s, i 0}-e_{s, j 0}, d_{r, i}=$ $d_{r, i 0}-d_{r, j 0}, j=i-1$, then the error dynamics are given as

$$
\begin{aligned}
& \dot{e}_{s, i 0}=e_{q, i 0}, \\
& \dot{e}_{q, i 0}=-b e_{q, i 0}-\left(k_{0}+k_{1}\right) e_{s, i 0}+k_{1} e_{s, j 0} .
\end{aligned}
$$

Let us define $e_{s, 0}=\left[e_{s, 10}, \ldots, e_{s, i 0}, \ldots, e_{s, N 0}\right]^{T}$ and $e_{q, 0}=$ $\left[e_{q, 10}, \ldots, e_{q, i 0}, \ldots, e_{q, N_{0}}\right]^{T}$ as the vectors of position and velocity errors, respectively. Thus, the error state vector, $\mathcal{X}(t)=\left[\begin{array}{ll}e_{s, 0} & e_{q, 0}\end{array}\right]^{T}$, is given as

$$
\dot{\mathcal{X}}(t)=A \mathcal{X}(t)
$$

where $A$ is given by $A=\left[\begin{array}{cc}O_{N} & I_{N} \\ -P & -D\end{array}\right], I_{n}$ and $O_{n}$ denote $(n \times n)$ identity and $(n \times n)$ null matrices, respectively, $D=\operatorname{diag}\{b, \ldots, b\} \in \mathbb{R}^{N \times N}$, and $P=\left[p_{i i}\right] \in \mathbb{R}^{N \times N}$ is represented by

$$
p_{i i}= \begin{cases}k_{0}, & j=i, i=1, \\ k_{0}+k_{1}, & j=i, i>1, \\ -k_{1}, & j=i-1, i>1, \\ 0, & \text { otherwise. }\end{cases}
$$

The eigenvalues of $P, \lambda_{P, i}$, are $\lambda_{P, 1}=k_{0}$ and $\lambda_{P, i}=k_{0}+$ $k_{1}, \forall i>1$. Thus, $\forall k_{0}>0$ and $k_{1}>0$, the matrix $P$ is positive stable. In addition, $\forall b>0$, the matrix $D$ is positive stable.

Exploiting the Schur's formula [16], the characteristics equation of $A$ is calculated as

$$
\operatorname{det}\left(S I_{2 N}-A\right)=\prod_{i=1}^{N}\left(S^{2}+b S+\lambda_{P, i}\right) \text {. }
$$

The polynomial $S^{2}+b S+\lambda_{P, i}$ is Hurwitz stable under the conditions $b>0, k_{0}>0, k_{1}>0$, i.e., the matrix $A$ is stable. Consequently, the dynamics are asymptotically stable i.e., $\lim _{t \rightarrow \infty} \mathcal{X}(t)=0$.

2) String Stability Analysis: To simplify our analysis, let us define $e_{i}=s_{j}-s_{i}-d_{r, i}, \dot{e}_{i}=q_{j}-q_{i}, \ddot{e}_{i}=u_{j}-u_{i}$, $e_{i 0}=s_{0}-s_{i}-d_{r, i 0}$, and $\dot{e}_{i 0}=q_{0}-q_{i}$.

Form (13) one can write $\ddot{e}_{i}$ as

$$
\begin{aligned}
\ddot{e}_{i} & =u_{j}-u_{i} \\
& =b \dot{e}_{j 0}+k_{0} e_{j 0}+k_{1} e_{j}-b \dot{e}_{i 0}-k_{0} e_{i 0}-k_{1} e_{i},
\end{aligned}
$$

Writing (18) in the S-domain results in

$$
H(S)=\frac{E_{i}(S)}{E_{j}(S)}=\frac{k_{1}}{S^{2}+b S+c},
$$

where $c=k_{0}+k_{1}$.
The $L_{\infty}$ string stability can be guaranteed by satisfying $\|h(t)\|_{1}<1$ and $h(t)>0$, where $h(t)=\mathcal{L}^{-1}\{H(S)\}$ is the impulse response of $H(S)$.

The poles of $H(S)$ are $p_{1,2}=-0.5 b \pm \sqrt{(0.5 b)^{2}-c}$. Therefore, to achieve $h(t)>0$, the response of $H(S)$ have to be over-damped, i.e., $(0.5 b)^{2}>c$, or to be critical-damped, i.e., $(0.5 b)^{2}=c$. However, for a higher speed response, the parameters of $H(S)$ are designed to achieve a criticaldamped response, i.e., $(0.5 b)^{2}=c$. In such as case, we have $h(t)=k_{1} t e^{-0.5 b t}$, so $\forall k_{1}>0$, we satisfy $h(t)>0$.

Lemma 1. [17]. If $G(s)$ is a stable proper transfer function with $|g(0)|=\delta$, and if $g(t)$ is its impulse response, then $\|g(t)\|_{1}=\delta$ if and only if $g(t)$ always has the same sign.

Exploiting Lemma 1, we have

$$
\|h(t)\|_{1}=|H(0)|=\frac{k_{1}}{c}=\gamma .
$$

Consequently, by setting $k_{1}=\gamma c$ with $\gamma<1$, then $\|h(t)\|_{1}<1$ and as a result the platoon is strong string stable.

3) Transient Response: Let us define the eigenvalues of the system matrix $A$ as $\lambda_{A, i}$, so from (17), $\lambda_{A, i}$ is given by

$$
\lambda_{A, i_{1,2}}=-0.5 b \pm \sqrt{(0.5 b)^{2}-\lambda_{P, i}}
$$

Comparing (21) with the standard form of the characteristic equation of second order system, then the settling time is $T_{s}=4 \tau=\frac{8}{b}$, damping factor $\zeta=\frac{b}{2 \sqrt{\lambda}}$.

4) Design of $u_{i}^{p}$ for Gap Closure Capability: As discussed in the transient analysis, the speed of the response can be designed from the relation, $c=\left(\frac{b}{2 \zeta}\right)^{2}$ with $c=k_{0}+k_{1}$ and $k_{1}=\gamma c$. Therefore, during the gap closing, if the error $e_{s, i}$ is larger than a certain value, $e_{u}$, smaller value for $\zeta, \zeta_{l}$, is allowed to increase the rising time and a higher weight is given for $e_{s, i}$ than that for $e_{s, i 0}$ via changing the value of $\gamma$ to $\gamma_{u}=1$. As soon as, the error approaches $e_{l}$ this will be an indication for the end of gap closure scenario and the value of $\zeta$ and $\gamma$ converge to the values required for string stability, $\zeta_{u}=1$ and $\gamma_{l}<1$, respectively. Consequently, a smooth commutation between the normal platooning mode and the gap closing mode can be done by the calculation of $\zeta$ and $\gamma$ as follows

$$
\zeta= \begin{cases}\zeta_{u}, & e_{s, i} \leq e_{l} \\ \zeta_{l}, & e_{s, i} \geq e_{u} \\ \frac{\zeta_{u}-\zeta_{l}}{2}\left(1+\cos \left(\pi \frac{e_{s, i}-e_{l}}{e_{u}-e_{l}}\right)\right)+\zeta_{l}, & \text { otherwise }\end{cases}
$$

$$
\gamma= \begin{cases}\gamma_{l}, & e_{s, i} \leq e_{l} \\ \gamma_{u}, & e_{s, i} \geq e_{u} \\ \frac{\gamma_{u}-\gamma_{l}}{2}\left(1+\cos \left(\pi \frac{e_{s, i}-e_{u}}{e_{u}-e_{l}}\right)\right)+\gamma_{l}, & \text { otherwise }\end{cases}
$$

To evaluate the gap closure scenario, it is required to quantify the corresponding time required to reduce the gap. 
Thus, the absolute error is integrated over time. For such performance evaluation, let us consider a discrete time span defined by $t \in\left[t_{0}, t_{K}\right]$ with equal segments, $K$, of length $\Delta t_{k}$.

$$
Q_{g c, i}=\sum_{k=1}^{K}\left|e_{s, i}(t)\right| \Delta t_{k}
$$

\section{B. Design of $u_{i}^{c}$}

In this part, the controller part $u_{i}^{c}$ is designed based on the artificial potential field that guarantees the collision avoidance of vehicle $i$ with its predecessor.

The $u_{i}^{c}$ can be designed for longitudinal collision avoidance as follows.

$$
u_{i}^{c}=-\nabla_{s_{i}} \varphi_{i}=-\frac{\partial \varphi_{i}}{\partial s_{i}},
$$

where $\varphi_{i}$ is the artificial potential field produced by vehicle $i$.

In the literature several potential field approaches have been studied to guarantee the collision avoidance of multiagent systems [11]. Let us define the potential field as

$$
\varphi_{i}=\frac{1}{\Gamma_{i}^{k_{c}}},
$$

where $d_{i}=s_{i-1}-s_{i}, k_{c}>0$ is a tuning parameter, and $\Gamma_{i}$ is the potential function that is adapted as

$$
\Gamma_{i}=\left(1-\alpha \frac{\left(\left|d_{i}\right|^{2}-d_{s}^{2}\right)^{2}}{1+\left(\left|d_{i}\right|^{2}-d_{s}^{2}\right)^{2}}\right)^{\rho}
$$

where, $d_{s}$ is the desired safe inter-vehicle distance and $\alpha=$ $\frac{1+d_{s}^{4}}{d^{4}} . \alpha$ is chosen such that the function $\Gamma_{i}$ is differentiable at $d_{s}$, so the gradient of $\varphi_{i}$ remains continuous everywhere. The value of $\Gamma_{i}$ lays in the interval $[0,1]$, becoming 0 when $d_{i}=0$, and 1 when $d_{i}>d_{s}$. Clearly, $\varphi_{i} \rightarrow \infty$ whenever $\Gamma_{i} \rightarrow 0$. Thus, moving in the direction of the negative gradient of $\varphi_{i}$ guarantees that the collisions between the cars will be avoided. The reason for choosing $\Gamma_{i}$ as in (27) is two fold. First, it is a smooth function that is the transition between none-collision and collision states is done smoothly. Second, it has local effect, i.e., the collision avoidance part is ineffective once the car is sufficiently far from the predecessor. The exponent $\rho$ is chosen as $\rho=\frac{1-\operatorname{sign}\left(\left|d_{i}\right|-d_{s}\right)}{2}$ to force $\Gamma_{i}$ to be constant beyond a distance $d_{s}$, so the collision avoidance part will only affect the value of control signal if $d_{i}$ approaches $d_{s}$.

The main result of this paper can be stated as follows.

Theorem 1. Consider the platoon system whose longitudinal model defined in (1), (2), (3) - (8), and (10), under assumptions 1 - 3, and the proposed control algorithm described in (11), (13), (22), (23), and (25) is applied. Then, with both gap closure and collision avoidance capabilities, the platoon tracking error convergence and string stability are guaranteed as long as, $\forall \gamma<1$, the following conditions are satisfied: $b>0, c=0.25 b^{2}, k_{1}=\gamma c$, and $k_{0}=(1-\gamma) c$.
Proof. Applying the control law (11) to the system (10), then the error dynamics (15) become

$$
\dot{\mathcal{X}}(t)=A \mathcal{X}(t)+B U^{c}
$$

where $B=\left[\begin{array}{c}O_{N} \\ I_{N}\end{array}\right]$ and $U^{c}=\left[-u_{1}^{c},-u_{2}^{c}, \ldots,-u_{N}^{c}\right]^{T}$.

There are two cases of operation, including normal case (i.e., no collision is detected $d_{i}>d_{s}$ ) in which $U^{c}=0$, and collision case in which $U^{c} \neq 0$. For the normal case and from the stability analysis presented in the previous sections, one can set the following conditions: $\forall \gamma<1, b>0, c=0.25 b^{2}$, $k_{1}=\gamma c$, and $k_{0}=(1-\gamma) c$, for both platoon asymptotic stability and string stability.

For the collision case $\left(d_{i}<d_{s}\right), U^{c} \neq 0$, so if one proves the boundedness of $U^{c}$, one can deduce that the platoon will be stable with steady state error, i.e., $\lim _{t \rightarrow \infty} \dot{\mathcal{X}}(t)=0$ and $\lim _{t \rightarrow \infty} \mathcal{X}(t) \neq 0$. Thus, next, we will prove that $U^{c}$ is bounded.

Let us recast (26) as

$$
\varphi_{i}=\left(\beta_{i}\right)^{-\rho k_{c}}
$$

where $\beta_{i}$ is given by

$$
\beta_{i}=1-\alpha \frac{\left(\left|d_{i}\right|^{2}-d_{s}^{2}\right)^{2}}{1+\left(\left|d_{i}\right|^{2}-d_{s}^{2}\right)^{2}} .
$$

Thus,

$$
\nabla_{s_{i}} \varphi_{i}=-\rho k_{c}\left(\beta_{i}\right)^{-\rho k_{c}-1} \nabla_{s_{i}} \beta_{i}
$$

From (30), $\left(\beta_{i}\right)^{-\rho k_{c}-1}$ is bounded provided that $\left|d_{i}\right| \neq 0$. in the rest of the proof will find the bound of $\nabla_{s_{i}} \beta_{i}$.

Let us define $w_{i}=\left|d_{i}\right|^{2}-d_{s}^{2}$, so (30) can be recast as

$$
\beta_{i}=1-\alpha \frac{w_{i}^{2}}{1+w_{i}^{2}} .
$$

Consequently, $\nabla_{s_{i}} \beta_{i}$ is given by

$$
\begin{aligned}
\nabla_{s_{i}} \beta_{i} & =\nabla_{w_{i}} \beta_{i} \nabla_{s_{i}} w_{i} \\
& =\frac{-2 \alpha w_{i}}{\left(1+w_{i}^{2}\right)^{2}} 2 \sqrt{w_{i}+d_{s}^{2}}
\end{aligned}
$$

From (33), one can deduce

$$
\left|\nabla_{s_{i}} \beta_{i}\right|<\left(1+w_{i}^{2}\right)^{2}\left|\nabla_{s_{i}} \beta_{i}\right| .
$$

The maximum value of the term $\left(1+w_{i}^{2}\right)^{2}\left|\nabla_{s_{i}} \beta_{i}\right|$ can be easily found using the conventional method which equals to $\frac{8 \alpha d_{s}^{3}}{3^{\frac{3}{2}}}$ at $w_{i}=\frac{-2}{3} d_{s}^{2}$. Thus, the value of $\left|\nabla_{s_{i}} \beta_{i}\right|$ is bounded. As a result, the control signal, $u_{i}^{c}$ is bounded and hence $U^{c}$ is bounded.

\section{RESUlTS}

\section{A. Environment}

The considered urban cars platoon with the proposed control strategy is simulated in ICARS framework. The platoon under study includes 3 follower vehicles (Model: Robotized Renault ZOE) plus a manually driven leader (Model: Renault FLUENCE electric equipped with sensors 
and communication module). The parameters of the vehicle is given in Table I. The vehicles are initially parked and we use the maps of Ecole Centrale de Nantes campus. The leader moves from its starting location to the destination points. During its motion its path is built online and sent via radio communication module to the followers vehicles as the reference path to follow. Radio system receives the vehicle information in the platoon one by one. Thus, an algorithm is developed in the ICARS framework to receive the information from the radio module and then synchronize them. The lateral controller proposed in [18] is responsible for producing the vehicle steering angle of enable them to follow the reference path of the leader by minimizing both the lateral and angular deviations. Results for the lateral motion are shown in Fig. 3. The longitudinal controller is

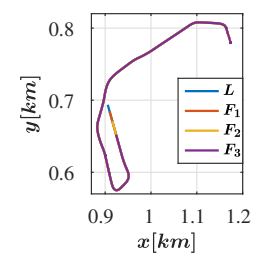

(a) Path Tracking

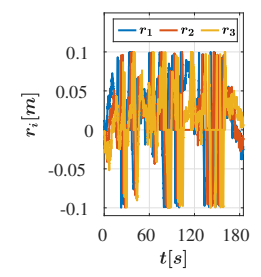

(b) $r_{i}$

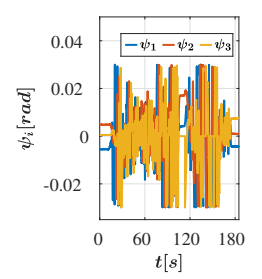

(c) $\psi_{i}$
Fig. 3: Lateral Control Results; L: Leader Actual Path, and $F_{i}$ : Follower $i$ Actual Path.

TABLE I: Vehicles parameters for the realistic study

\begin{tabular}{|c|c|c|}
\hline Parameter/Vehicle & FLUENCE & ZOE \\
\hline Mass & $1605 \mathrm{~kg}$ & $1428 \mathrm{~kg}$ \\
\hline Width & $1.545 \mathrm{~m}$ & $1.945 \mathrm{~m}$ \\
\hline Height & $1.462 \mathrm{~m}$ & $1.562 \mathrm{~m}$ \\
\hline Length & $4.748 \mathrm{~m}$ & $4.084 \mathrm{~m}$ \\
\hline Rear-axis to Rear-end & $1.140 \mathrm{~m}$ & $0.657 \mathrm{~m}$ \\
\hline distance between axes & $2.701 \mathrm{~m}$ & $2.588 \mathrm{~m}$ \\
\hline distance between wheels & $1.545 \mathrm{~m}$ & $1.511 \mathrm{~m}$ \\
\hline Wheel radius & $0.29 \mathrm{~m}$ & $0.29 \mathrm{~m}$ \\
\hline Inertia momentum & $45.0 \mathrm{kgm}^{2}$ & $28.0 \mathrm{kgm}^{2}$ \\
\hline
\end{tabular}

implemented at a rate of $100 \mathrm{~Hz}$. Control parameters, given in Table II, are chosen such that both the internal stability and string stability are guaranteed.

TABLE II: Controller Parameters

\begin{tabular}{|c|c|c|c|}
\hline Parameter & Value & Parameter & Value \\
\hline$u \in$ & {$[-6,1] \mathrm{m} / \mathrm{s}^{2}$} & $v \in$ & {$[0,8] \mathrm{m} / \mathrm{s}$} \\
\hline$d_{r, i}$ & $10 \mathrm{~m}$ & $\gamma$ & 0.5 \\
\hline$e_{l}$ & $0.2 d_{r, i}$ & $e_{u}$ & $0.8 d_{r, i}$ \\
\hline$\zeta_{l}$ & 0.001 & $b$ & 1.6 \\
\hline$k_{c}$ & 1.5 & $d_{s}$ & $5 \mathrm{~m}$ \\
\hline
\end{tabular}

\section{B. Normal Platooning Scenario}

In this study, the leader travels with variable velocity. The vehicles are initially parked in different positions such that the initial relative distances different from desired ones. Fig.
4 illustrates the results for the leader tracking consensus. The vehicles can achieve the consensus and the Curvilinear position and speed errors converge. Results enlighten the capability of the proposed strategy to create and maintain the platoon in case of a human-driven leader (i.e., the velocity is variable). Table III illustrates the Root Mean Squared Error (RMSE) of the tracking errors which is in the acceptable ranges. Furthermore, a sudden break of the leader occurs

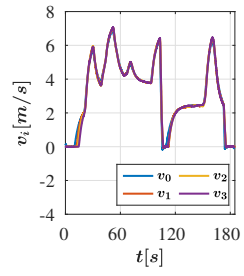

(a) $v_{i}$

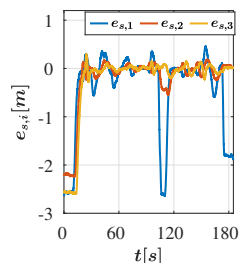

(b) $e_{s, i}$

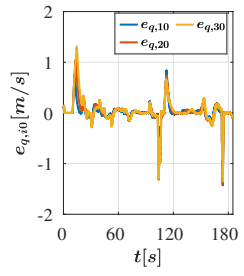

(c) $e_{q, i 0}$
Fig. 4: Platoon maintaining scenario.

at instant $100 \mathrm{~s}$ and it smoothly stops at instant $170 \mathrm{~s}$. The results in Fig. 4, show that the position errors of the vehicles are attenuated as $e_{s, 1}=-2.6 \mathrm{~m}, e_{s, 2}=-0.5 \mathrm{~m}$, and $e_{s, 3}=-0.2 \mathrm{~m}$, which enlightens the string stability of the platoon.

TABLE III: RMSE of tracking errors

\begin{tabular}{|c|c|c|c|}
\hline RMSE / Follower $i$ & 1 & 2 & 3 \\
\hline$e_{s, i}[m]$ & 0.2103 & 0.0872 & 0.0482 \\
\hline$e_{q, i}[\mathrm{~m} / \mathrm{s}]$ & 0.0763 & 0.0297 & 0.0219 \\
\hline
\end{tabular}

\section{Gap Closure Scenario}

To investigate the integrated controller capability in case of a gap closing scenario, follower 3 starts at a $32 \mathrm{~m}$ gap from its desired set point while the other followers move in normal platoon. As soon as the leader sends commands to the follower 3 to join the platoon, it will move in a gap closing mode, as $e_{e, 3}>e_{l}$, until it reaches a position where $e_{e, 3}<=e_{l}$, then it moves in normal platoon.

Fig. 5 shows the numerical results for a gap closing scenario. These results show that the proposed algorithm is able to reduce the gap closing time without violates the acceleration and velocity constraints for both passenger comfortability and safety. To quantify these results, one can use the performance index (24) which ensures that the response is improved by $11.4 \%$.

\section{Collision Avoidance Scenario}

In this scenario, we test how a vehicle reacts in case of a sudden braking of a front vehicle inside the platoon. A braking maneuver performed by the follower 1 where its speed is sharply reduced at instant $45 \mathrm{~s}$ from $5.9 \mathrm{~m} / \mathrm{s}$ to a full stop. Results in Fig. 6 show how the vehicle performs in case that collision avoidance controller is not used, i.e., $u_{i}^{c}=0$. These results illustrate that platoon is failed and collision occurs between vehicles 2 and 1 . 


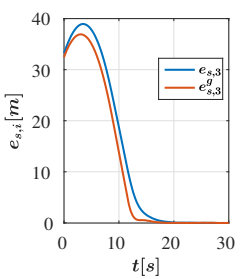

(a) $e_{s, 3}$

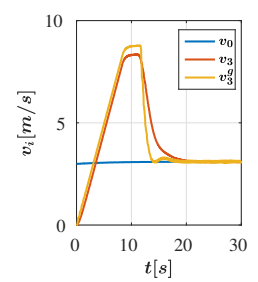

(b) $v_{3}$

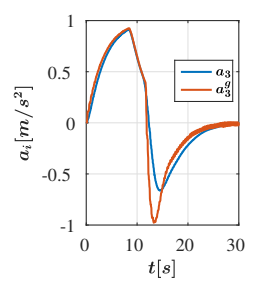

(c) $a_{3}$
Fig. 5: Demonstration of Gap Closure Scenario for the $3^{\text {rd }}$ vehicle: comparison study. $(\cdot)^{g}$ value in case of using the Gap closure algorithm

On the other hand, the results show how the platoon reacts in case that collision avoidance controller is integrated in the control law. As shown in the results, as soon as the intervehicle distance violates the safety region $\left(d_{i}>d_{s}\right)$, the collision avoidance control is activated and it increases in the negative direction to stop the vehicle such that a safe distance $d_{s}=5 \mathrm{~m}$ can be guaranteed. The simulations are demonstrated in this video.

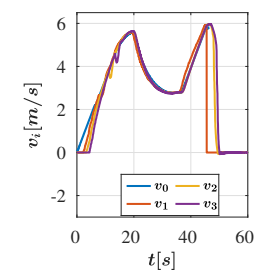

(a) $v_{i}$

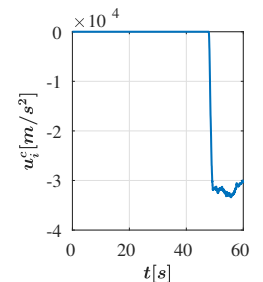

(b) $u_{2}^{c}$

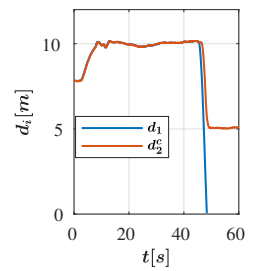

(c) $d_{2}$
Fig. 6: Demonstration of collision avoidance capability: comparison study. $(\cdot)^{c}$ denotes the value in case of using the collision avoidance capability

\section{CONCLUSIONS}

A unified consensus-based control technique has been proposed to address different issues of platooning in urban environment, including normal platooning, gap closure, and collision avoidance. A second-order longitudinal model of the platoon in the path coordinates is presented. The controller is designed to achieve convergence of the tracking errors taking into consideration the motion in urban environments. An algorithm for adapting the controller parameters is presented to achieve gap closure scenario. Furthermore, a collision avoidance algorithm is designed and integrated with the main controller to guarantee a safety region between vehicles during their travel in the platoon. Conditions to guarantee internal stability and string stability are given. The resulting algorithm was implemented in a realistic simulator called ICARS with real vehicles models. Simulation results have validated the proposed strategy and the theoretical work.

\section{ACKNOWLEDGMENTS}

The work presented in this paper is accomplished in the framework of the Valet project (reference: ANR-15-CE220013-02).

\section{REFERENCES}

[1] A. Khalifa, O. Kermorgant, S. Dominguez, and P. Martinet, "Vehicles platooning in urban environment: Consensus-based longitudinal control with limited communications capabilities," in International Conference on Control, Automation, Robotics and Vision, 2018.

[2] F. Lin, M. Fardad, and M. R. Jovanovic, "Optimal control of vehicular formations with nearest neighbor interactions," IEEE Transactions on Automatic Control, vol. 57, no. 9, pp. 2203-2218, 2012.

[3] S. Santini, A. Salvi, A. S. Valente, A. Pescapé, M. Segata, and R. L. Cigno, "A consensus-based approach for platooning with intervehicular communications and its validation in realistic scenarios," IEEE Transactions on Vehicular Technology, vol. 66, no. 3, pp. 19851999, 2017.

[4] H. Chehardoli and M. R. Homaeinezhad, "Third-order leaderfollowing consensus protocol of traffic flow formed by cooperative vehicular platoons by considering time delay: constant spacing strategy," the Journal of Systems and Control Engineering, vol. 232, no. 3, pp. 285-298, 2018.

[5] S. E. Li, H. Peng, K. Li, and J. Wang, "Minimum fuel control strategy in automated car-following scenarios," IEEE Transactions on Vehicular Technology, vol. 61, no. 3, pp. 998-1007, 2012.

[6] J. VanderWerf, S. Shladover, N. Kourjanskaia, M. Miller, and H. Krishnan, "Modeling effects of driver control assistance systems on traffic," Transportation Research Record: Journal of the Transportation Research Board, no. 1748, pp. 167-174, 2001.

[7] M. Fardad, F. Lin, and M. R. Jovanović, "Sparsity-promoting optimal control for a class of distributed systems," in American Control Conference (ACC), 2011. IEEE, 2011, pp. 2050-2055.

[8] F. Gao, S. E. Li, Y. Zheng, and D. Kum, "Robust control of heterogeneous vehicular platoon with uncertain dynamics and communication delay," IET Intelligent Transport Systems, vol. 10, no. 7, pp. 503-513, 2016.

[9] Y. Wu, S. E. Li, Y. Zheng, and J. K. Hedrick, "Distributed sliding mode control for multi-vehicle systems with positive definite topologies," in IEEE $55^{\text {th }}$ Conference on Decision and Control (CDC). IEEE, 2016, pp. 5213-5219.

[10] W. B. Dunbar and D. S. Caveney, "Distributed receding horizon control of vehicle platoons: Stability and string stability," IEEE Transactions on Automatic Control, vol. 57, no. 3, pp. 620-633, 2012

[11] M. M. Zavlanos and G. J. Pappas, "Potential fields for maintaining connectivity of mobile networks," IEEE Transactions on Robotics, vol. 23, no. 4, pp. 812-816, 2007.

[12] P. Shen, H. Zou, X. Zhang, Y. Li, and Y. Fang, "Platoon of autonomous vehicles with rear-end collision avoidance through time-optimal pathconstrained trajectory planning," in $11^{\text {th }}$ International Workshop on Robot Motion and Control (RoMoCo). IEEE, 2017, pp. 232-237.

[13] A. Ferrara and C. Vecchio, "Collision avoidance strategies and coordinated control of passenger vehicles," Nonlinear Dynamics, vol. 49, no. 4, pp. 475-492, 2007.

[14] Y. Kuriki and T. Namerikawa, "Consensus-based cooperative formation control with collision avoidance for a multi-uav system," in American Control Conference (ACC), 2014. IEEE, 2014, pp. 2077 2082.

[15] S. E. Li, Y. Zheng, K. Li, and J. Wang, "An overview of vehicular platoon control under the four-component framework," in Intelligent Vehicles Symposium (IV), 2015 IEEE. IEEE, 2015, pp. 286-291.

[16] Y. Cao, W. Ren, and Y. Li, "Distributed discrete-time coordinated tracking with a time-varying reference state and limited communication," Automatica, vol. 45, no. 5, pp. 1299-1305, 2009.

[17] S. Darbha, "String stability of interconnected systems: An application to platooning in automated highway systems," Ph.D. dissertation, University of California, 1994.

[18] B. Thuilot, J. Bom, F. Marmoiton, and P. Martinet, "Accurate automatic guidance of an urban electric vehicle relying on a kinematic gps sensor,' IFAC Proceedings Volumes, vol. 37, no. 8, pp. 155-160, 2004. 